Pflegeexpertin APN in der Kinderkardiologie

\title{
Über die Schulter geschaut
}

Im Rahmen des Masterstudiums in Pflegewissenschaft hatte Gabriela Staub die Möglichkeit, eine Pflegeexpertin „Advanced Practice Nurse Kardiologie“ im Universitäts-Kinderspital Zürich während eines Workshadowings zu begleiten. Diese Art Praktikum ist gut geeignet, um Einblick in den Tätigkeitsbereich APN zu erlangen und so die eigene Rollenbildung zu fördern.

Gabriela Staub, Daniela Händler-Schuster, Romy Mahrer Imhof

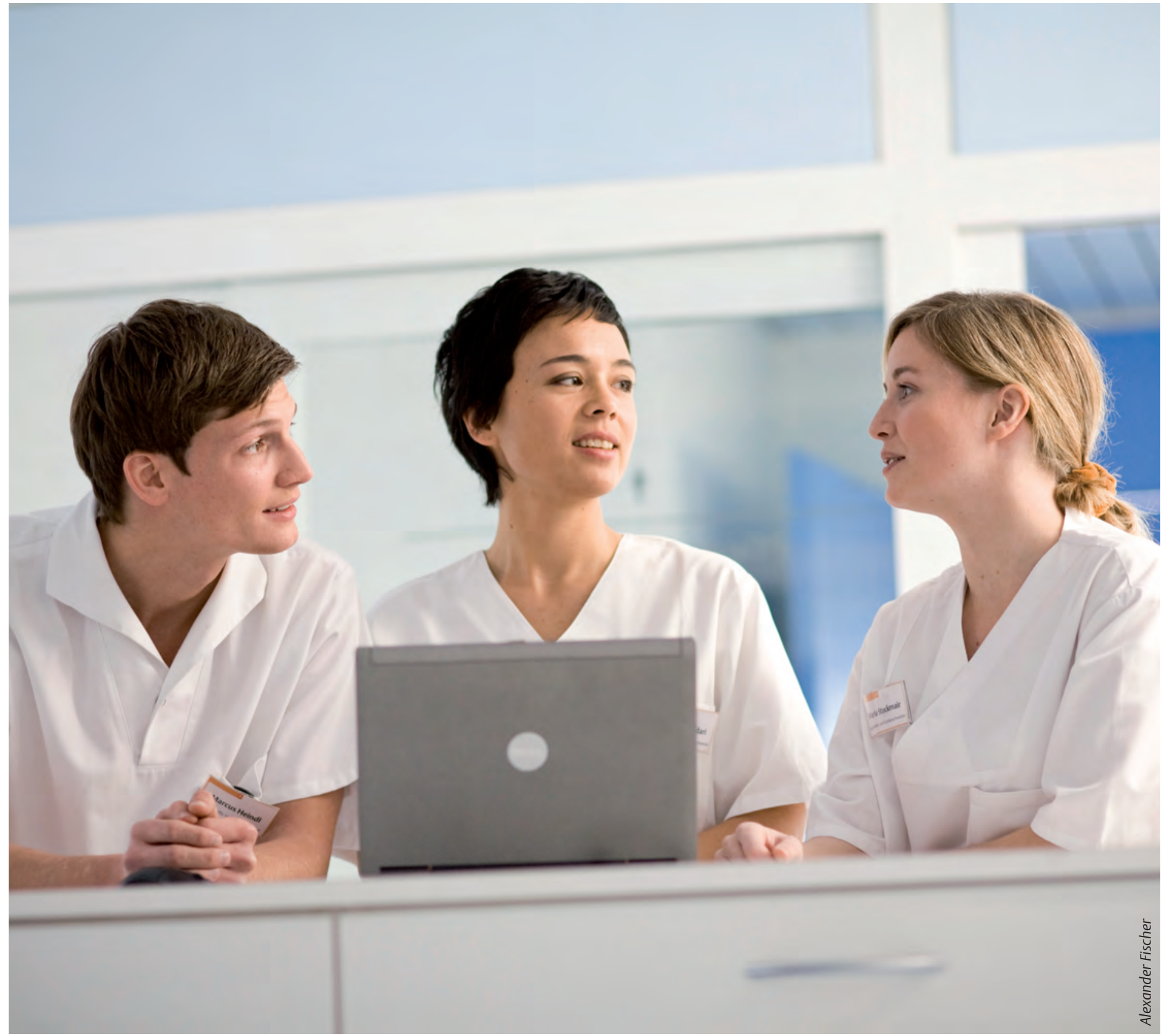


$\square$

Das Universitäts-Kinderspital Zürich ist das größte pädiatrische Herzzentrum in der Schweiz. Kinder ab dem Neugeborenenalter bis zum adoleszenten Patienten werden dort betreut. Jährlich erhalten ca. 350 Kinder im eigenen pädiatrischen Herzkatheterlabor einen diagnostischen oder interventionellen Eingriff und weitere ca. 350 Kinder werden pro Jahr am Herzen operiert. Darunter sind auch Kinder mit einer schweren Kardiomyopathie. Studien konnten aufzeigen, dass Familien mit einem an Kardiomyopathie erkrankten Kind aufgrund des Schweregrads der Erkrankung emotional stärker belastet sind als Familien mit einem Kind, das an einer anderen kardiologischen Erkrankung leidet. ${ }^{1}$ Die Erstautorin hatte das Ziel, einen Einblick in das Tätigkeitsfeld einer APN zu erhalten. Das persönliche Ziel war, durch das Workshadowing die eigene Rollenbildung zu fördern.

Dieser Beitrag beginnt mit der Begriffsdefinition APN. Zur besseren Darstellung der Aufgaben einer APN und um den Mehrwert aufzuzeigen, den Familien durch das Konzept von APN erhalten, dient das Beispiel eines kleinen Patienten, der an einer Kardiomyopathie erkrankt ist. Es folgt die Betrachtung der Vorteile des Konzepts und die Analyse, wer im Umfeld des Spitals die Befürworter zur Umsetzung der APN-Rolle sind und wer mit welchen Argumenten noch überzeugt werden muss.

\section{Definition und Aufgaben einer APN}

Der Begriff APN steht für ein Schirmkonzept, dem sich unterschiedliche Rollen einer APN zuweisen lassen. Der International Council of Nurses (ICN) definiert eine APN als registrierte Pflegefachperson, die sich Expertenwissen, Fähigkeiten für komplexe Entscheidungsfindung sowie klinische Kompetenz für erweiterte Pflegepraxis angeeignet hat. Die Eigenschaften werden vom Kontext und/oder dem Land, in dem sie zur Ausübung der Tätigkeit zugelassen ist, geprägt. Ein Masterabschluss (Master in Nursing Science) gilt als Voraussetzung (ICN, 2009).

Die Aufgaben einer APN orientieren sich an dem Modell von Hamric et al. ${ }^{2}$ ( $\rightarrow$ Abb. 1). Sie unterteilen das Tätigkeitsfeld einer APN in Primärkriterien, die die Ausbildung betreffen, die Zentralkompe-

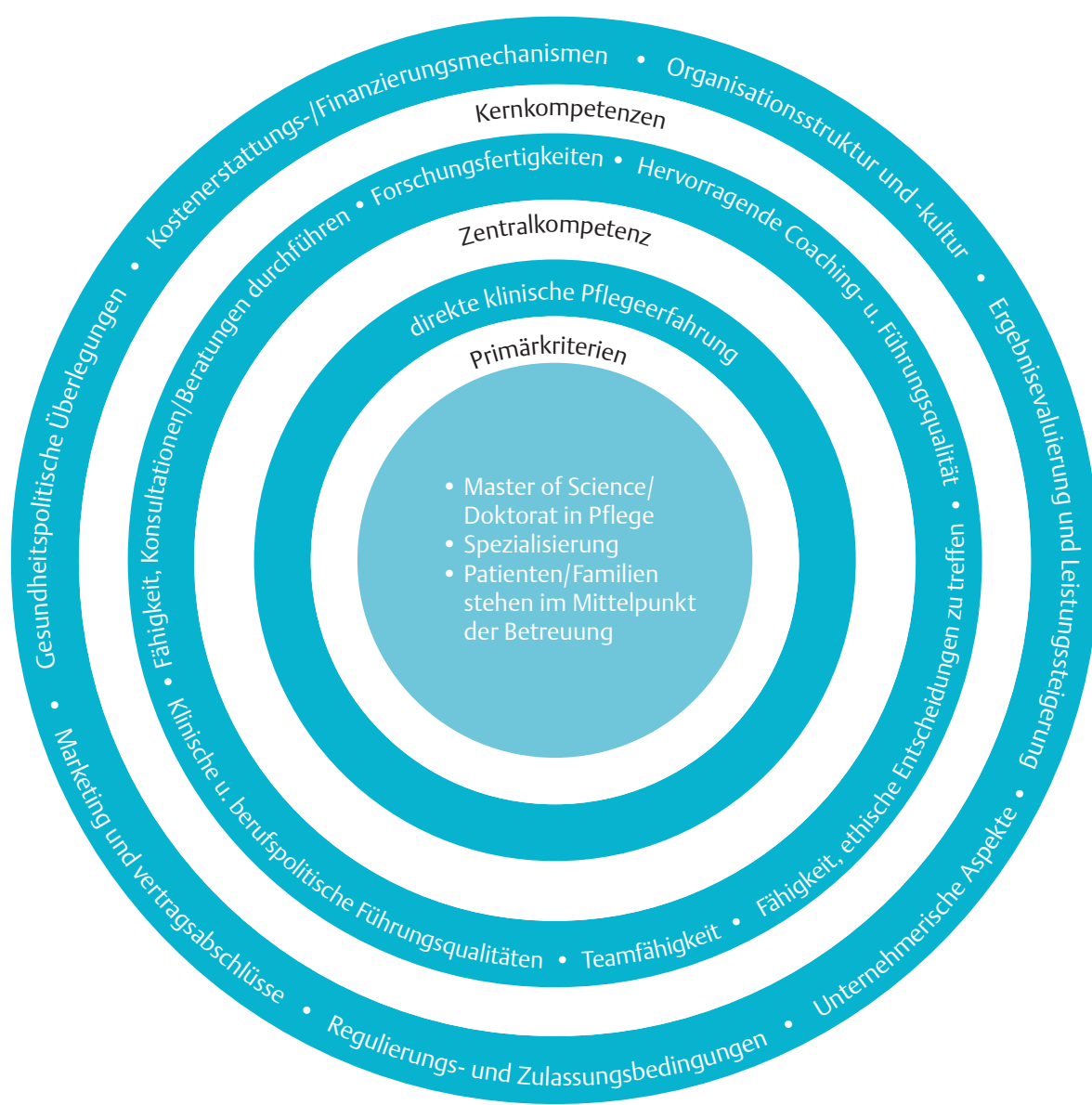

Abb. 1 APN-Modell.2

tenz, die die direkte klinische Praxis umfasst, sowie die sechs Kernkompetenzen: Coaching und Führung, Beratung und Konsultation, Forschungsfähigkeiten, klinisches und professionelles Leadership, Zusammenarbeit sowie ethische Entscheidungsfindung. Anhand eines Fallbeispiels werden die sechs Kernkompetenzen aufgeführt und erläutert, wie sich deren Umsetzung in die Praxis anhand des Fallbeispiels gestaltet.

\section{Advanced Nursing Practice (ANP) im Universitäts-Kinderspital Zürich - Befürworter in der Institution}

Am Universitäts-Kinderspital Zürich hat die Weiterentwicklung der Pflegepraxis durch den Einsatz von APN und ANP-Projekten seit 2002 eine große Bedeutung. Im Jahr 2002 startete das erste Projekt. Im Verlauf der Jahre kamen immer mehr Fachgebiete dazu. Es gibt verschiedene Optionen, ANP in die Praxis umzusetzen. In der Regel sind es Einzelpersonen mit einer akademischen
Ausbildung, die sich auf ein Fachgebiet spezialisiert haben. Im Kinderspital kommt der Teamansatz zum Tragen, der dadurch gekennzeichnet ist, dass sich eine Gruppe von Pflegenden unter der Leitung einer APNFachperson zusammenschließt und sich auf ein Fachgebiet spezialisiert. Seit rund zehn Jahren wird in verschiedenen Spezialgebieten das pflegerische Angebot für Familien systematisch entwickelt und evaluiert. Es besteht eine große Offenheit innerhalb der Institution für die APN-Rolle. Insbesondere die Pflegedirektorin und Leiterin Pflegeentwicklung nehmen mit der Etablierung von APNs im Universitäts-Kinderspital Zürich eine Pionierrolle in der Schweiz ein. Auf nationaler Ebene sind der Schweizerische Verein für Pflegewissenschaft sowie Swiss APN, aber auch die Elternvereinigungen für angeborene Herzfehler wichtige Schlüsselinstitutionen, die das Vorantreiben der Rollenentwicklung bzw. die Rollenetablierung befürworten. 


\section{Definition Kardiomyopathie}

Eine Kardiomyopathie bei Kindern und Jugendlichen ist eine sehr seltene, jedoch schwerwiegende Funktionsstörung des Herzmuskels. Die jährliche Inzidenz wird gemäß dem Kardiomyopathieregister der USA auf 1,13 Fälle pro 100.000 Personen unter 18 Jahren geschätzt. ${ }^{3}$ Die Kardiomyopathie wird in verschiedene Formen unterteilt, wobei die dilatative Kardiomyopathie (DCM) und die hypertrophe Kardiomyopathie (HCM) am häufigsten vorkommen. Bei beiden Formen handelt es sich um eine lebensbedrohliche Erkrankung, die mit einer abnormalen Herzstruktur und Herzfunktion sowie schlechten Prognosen einhergeht. ${ }^{4}$ Die Erkrankung ist sowohl die häufigste Ursache einer schweren Herzinsuffizienz, als auch die häufigste Indikation für eine Herztransplantation bei Kindern unter einem Jahr. ${ }^{4}$ Wie sich die Erkrankung zeigen kann, wird am folgenden Fallbeispiel beschrieben.

\section{Fallbeispiel}

Micha* ist sechs Jahre alt und das erste Kind einer Familie mit Migrationshintergrund. Bis vor seiner Erkrankung war er ein aktiver, gesunder Knabe. Die ersten Anzeichen der Erkrankung waren Zahnschmerzen, Appetitlosigkeit, Schwäche und Fieber. Kurze Zeit später erfolgte bei Atemnot eine Konsultation beim Kinderarzt. Wegen Verdachts auf eine obstruktive Bronchitis wurde eine Inhalationstherapie mit Ventolin angeordnet. Im Verlauf zeigte er zunehmend nächtliches Erwachen mit Aufsitzen, weiterhin Dyspnoe sowie schmerzhafte Bauchumfangszunahme. Nach Schwellung der Beine sowie des Skrotums und Bauchschmerzen wurde er im Spital des Wohnorts der Familie vorstellig. Dort wurde die Diagnose einer dilatativen Kardiomyopathie gestellt und es erfolgte eine unverzügliche Zuweisung ins Universitäts-Kinderspital Zürich. Rund zwei Monate später wurde wegen ventrikulärer Tachykardien sowie zur kardialen Resynchronisationstherapie die Implantation eines Implantable Cardiac-device (ICD) notwendig. Bei terminaler Herzinsuffizienz trotz maximaler konservativer und medikamentöser Herzinsuffizienz-

\footnotetext{
* Name geändert
}

therapie erfolgte sechs Wochen später die elektive Implantation eines biventrikulären Berlin Hearts (kardiales Ersatzverfahren) zur Überbrückung bis zur Transplantation. Rund vier Monate später wurde für Micha ein passendes Herz gefunden und er wurde erfolgreich transplantiert. Sein gesamter Aufenthalt im Universitäts-Kinderspital dauerte acht Monate, bis er in einem guten Allgemeinzustand nach Hause entlassen werden konnte.

Nachfolgend wird anhand der sechs Kernkompetenzen nach Hamric et al. ${ }^{2}$ das Tätigkeitsfeld der APN mit dem geschilderten Fallbeispiel erläutert.

\section{Coaching und Führung}

Diese Kompetenzen beinhalten die Patienten- respektive Familienedukation im stationären und ambulanten Setting. Zum Zeitpunkt der Diagnosestellung waren die Eltern mit unterschiedlichen Gefühlen konfrontiert, die als Zeit der Transition bezeichnet werden. Die wohl wichtigste Aufgabe war, die Familie durch diese Transition zu begleiten. Aufgrund der Erkrankung stand das Management der schweren Herzinsuffizienz zu Beginn im Vordergrund. Die Aufgabe der APN bestand unter anderem darin, die Eltern zu schulen. Nach der Herztransplantation nahmen Vorsichtsmaßnahmen und Pflegerichtlinien für transplantierte Kinder einen hohen Stellenwert in der Edukation ein. Die Eltern wurden von der APN Kardiologie in der Verabreichung von Medikamenten und Maßnahmen zur Hygiene angeleitet und somit befähigt, das aufwendige Therapiemanagement daheim weiterzuführen. Unter Berücksichtigung der Umgebungsfaktoren, aktueller Forschungsresultate und der Expertise der APN wurde stets darauf geachtet, dass die Präferenzen von Micha und seiner Familie respektiert wurden, wie beispielsweise Essgewohnheiten des Heimatlandes.

\section{Beratung und Konsultation}

Insbesondere als Micha mit dem Berlin Heart nach dem Intensivaufenthalt auf der Pflegestation betreut wurde, musste das Pflegeteam im Umgang mit dem Berlin Heart geschult werden. Für das Team war es eine besondere Situation, ein Kind, dessen Leben von diesem Gerät abhängig war, auf der Bettenstation zu haben. Die APN ver- fasste in interdisziplinärer Zusammenarbeit einen Leitfaden zur Betreuung des Patienten, Handhabung des Geräts sowie der Durchführung des Verbandwechsels und schulte die Pflegenden am Patientenbett. Mit der Zeit war es für Micha auch möglich, mit dem Berlin Heart im Spital spazieren zu gehen. Durch ihre Verfügbarkeit bei pflegerischen Fragen und spontanen Schulungen übernahm die APN eine wichtige Rolle, das Empowerment der Pflegenden zu fördern. Vor Austritt nach der Herztransplantation mussten die nachbetreuenden Institutionen beraten werden. Ein weiterer Aspekt dieser Kernkompetenz ist der Austausch bzw. das Teilen von persönlichem Wissen und Expertise mit anderen APNs und Gesundheitsfachpersonal. Die nationale und internationale Vernetzung der APN ist zentral, um Fachexpertise weiterentwickeln und Praxisfragen gemeinsam diskutieren zu können. Dies unterstützt wiederum die pflegerische Betreuung des Patienten und seiner Familie und führt zu einem besseren Patientenergebnis.

\section{Forschungsfähigkeiten}

Der Bedarf, Forschungsresultate im Sinne einer „best Evidence-based Practice“ in der Praxis zu generieren und zu implementieren, ist insbesondere bei seltenen Erkrankungen zentral. Durch die Literatursuche in relevanten Datenbanken zu pflegerischen Themen und der Bedeutung einer Kardiomyopathie für die Kinder und deren Eltern hat die APN einen wichtigen Beitrag geleistet, um für Micha und seine Familie eine Begleitung nach neusten Standards zu bieten. Dabei wurden aktuelle Forschungsergebnisse für Betreuungskonzepte für Kinder, die von einer Kardiomyopathie betroffen sind, sowie die Begleitung ihrer Familien kritisch beurteilt. Da es leider nur sehr wenige Studien in diesem Bereich gibt, unterstützt die APN nun ein sehr interessantes und praxisrelevantes Forschungsprojekt, um Daten für die Begleitung von Familien nach Neudiagnose einer Kardiomyopathie zu generieren - mit dem Ziel, betroffene Kinder und ihre Familien umfassender unterstützen und begleiten zu können.

Klinisches und professionelles Leadership Klinisches Leadership fokussiert sich zuerst auf den Patienten und seine Bedürf- 


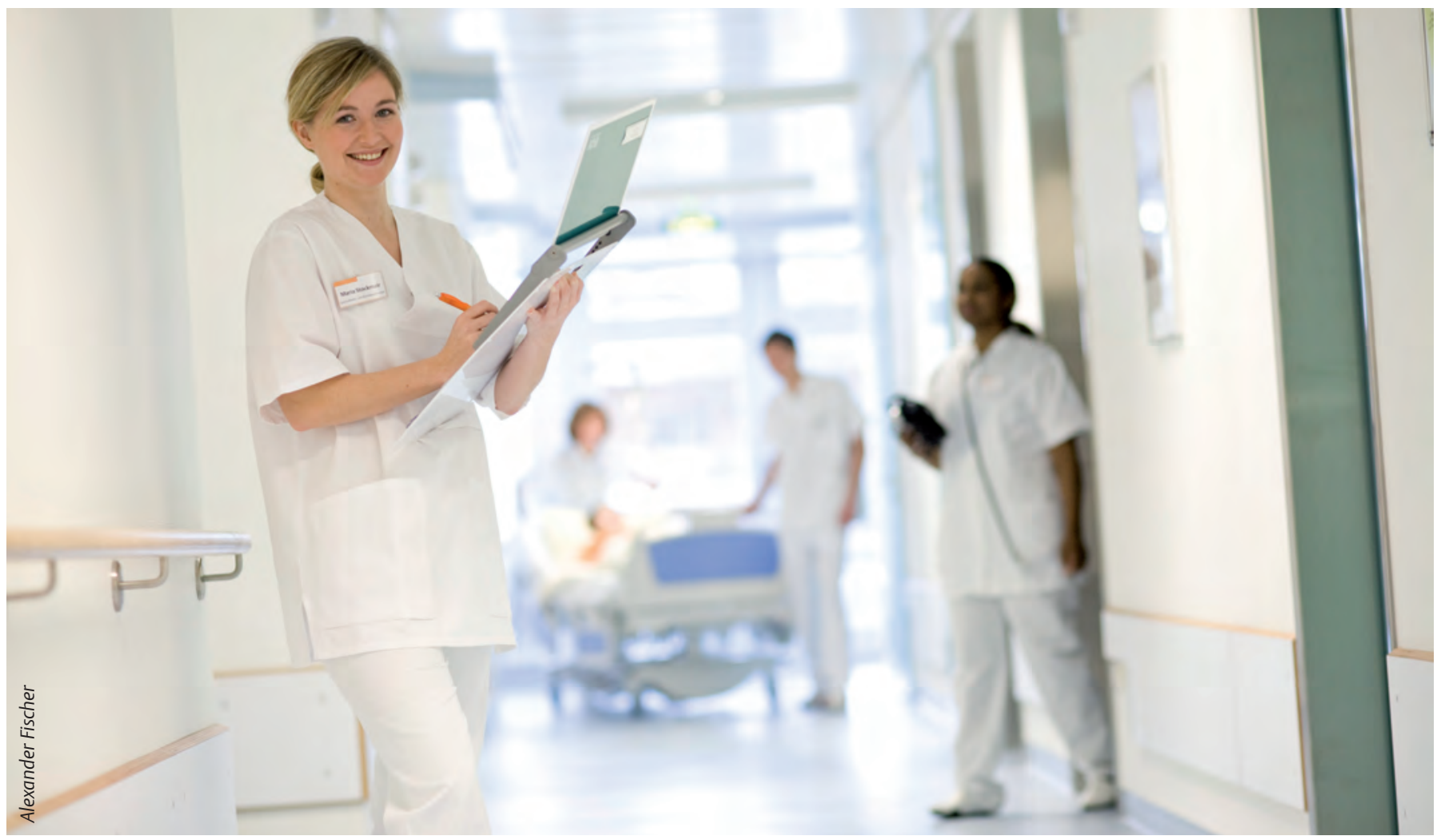

nisse. Die APN hat sichergestellt, dass die Pflege und Betreuung von Micha und seiner Familie von ausgezeichneter Qualität war. Dafür organisierte sie Fallbesprechungen und Schulungen zu relevanten pflegerischen Themen, die das Team beschäftigte. Um Leadership leben zu können, ist es wichtig, die Institution als Mikrosystem und das Makrosystem, welches das Mikrosystem beeinflusst, zu verstehen. ${ }^{2}$ Aufgrund der langjährigen Arbeitstätigkeit der APN Kardiologie im UniversitätsKinderspital Zürich kennt sie die Institution als Mikrosystem sehr gut und konnte deshalb einschätzen, welche pflegerischen Innovationen nötig waren, um Micha und seine Familie umfassend unterstützen zu können. Außerdem wusste sie, wer in der Institution an Bord geholt werden musste, damit Veränderungen reibungslos implementiert werden konnten. Zudem hatte das Mentoring und Empowerment der Familie sowie des Gesundheitspersonals einen wichtigen Stellenwert. Die APN übernahm dadurch eine Schlüsselrolle in der Betreuung und hat die Interessen des Patienten und seiner Familie vertreten. Bei Unklarheiten wurde sie durch Mitglieder des Behandlungsteams kontaktiert und koordinierte sowohl die Termine als auch die Begleitung der Familie und des involvierten Teams. Ferner konnte durch das professionelle Leadership der APN ein Arbeitsklima entstehen, das durch Vertrauen geprägt war.

\section{Zusammenarbeit}

In medizinisch sehr komplexen Situationen ist die multiprofessionelle Zusammenarbeit ein zentraler Aspekt, da sie die pflegerische Betreuung maßgeblich beeinflusst und durch gemeinsame Zielsetzungen bessere Patientenergebnisse ermöglicht werden. Einerseits ist die Zusammenarbeit mit dem Patienten und seinen Eltern zentral, aber auch die Zusammenarbeit mit den Pflegeteams, den ärztlichen und interdisziplinären Diensten ist äußerst wichtig. In die Betreuung von Micha war ein großes interdisziplinäres Betreuungsteam involviert. Ernährungsberatung, Physiotherapie, Psychologie, Kindergarten, Sozialdienst, Kardiopoliklinik, ärztlicher Dienst etc. haben alle einen wichtigen Beitrag zur Realisierung eines optimalen Therapiemanagements geleistet. Für die APN hat dies bedeutet, Termine mit zu koordinieren und dafür zu sorgen, dass der Informationsfluss aufrecht erhalten bleibt. Um dies zu erreichen, organisierte sie in regelmäßigen Abständen interdisziplinäre Sitzungen. Jedoch nicht nur Dienste der Institution, sondern auch externe Partner wie beispielsweise das Pflegefachpersonal, das die pflegerische Nachbetreuung im häuslichen Umfeld nach Austritt übernahm, musste frühzeitig involviert werden. Um diese Nachbetreuung sicherzustellen, wurden diese externen Partner mit detaillierten Pflegeplanungen über die Pflege von Micha informiert und ein telefonischer Kontakt hergestellt.

\section{Ethische Entscheidungsfindung}

Die Diagnose einer Kardiomyopathie und die Therapie im Verlauf können verschiedene ethische Fragen aufwerfen. Aufgrund des kulturellen Hintergrunds der Familie war die APN gefordert, im Rahmen von Fallbesprechungen ethische Konflikte zu erkennen und zu thematisieren. Anlass für Diskussionen gab insbesondere die Kommunikation innerhalb der Familie, die auf die Kultur zurückzuführen war. Es war 
eine Herausforderung, die Eltern davon zu überzeugen, dass eine offene Kommunikation mit Micha bezüglich der Therapie und der bevorstehenden Herztransplantation wichtig ist. Das Kommunikationsverständnis der Familie war teilweise für das Behandlungsteam schwierig einzuordnen, weil es sich von demjenigen in unserer Kultur unterschied. Durch zahlreiche Gespräche mit den Eltern und dem Behandlungsteam hat die APN versucht, für alle Beteiligten einen gangbaren Weg zu finden und dennoch den kulturellen Hintergrund der Familie zu respektieren.

\section{Mehrwert für Kinder, Jugendliche und Eltern}

Mit der Einführung von APN seit dem Jahr 2002 liegen die größten und wichtigsten Veränderungen für die Kinder, Jugendlichen und deren Familien darin, dass die Familien durch die APN-Tätigkeit eine wichtige und verlässliche Bezugsperson und einen Ansprechpartner haben, was zuvor nicht gegeben war. Der Fall von Micha in Bezug auf die Kernkompetenzen macht deutlich, dass Betroffene und ihre Familien von dem Handlungsfeld einer APN profitieren, indem eine Kontinuität gesichert ist. Besonders nach Spitalaustritt können sich heute Familien jederzeit bei Fragen oder Unklarheiten bei der zuständigen APN melden. Insbesondere in sehr komplexen Situationen übernimmt die APN auch nach Spitalaustritt eine wichtige Funktion, indem sie für die Weiterführung des Behandlungskonzepts die „Fäden in der Hand“ hält. Das Handlungsfeld einer APN ist für die Patienten und ihre Familien von großem Wert, weil dadurch die Kontinuität der Begleitung langfristig gewährleistet bleibt und die Betreuung an Qualität gewinnt.

\section{$\rightarrow$ FAZIT}

Das Ziel dieses Workshadowings war es für die Erstautorin, Einblick in das Tätigkeitsfeld einer APN zu erhalten und die persönliche Rollenfindung zu fördern. Es war eine sehr spannende Zeit. Die Erstautorin konnte die Bedeutung und Wichtigkeit dieser Rolle erkennen. Deutlich wurden besonders die Herausforderungen, die mit der Rollenumsetzung verbunden sind. Die Skepsis des ärztlichen Dienstes, aber auch des Pflegepersonals könnte die Weiterentwicklung der Rolle behindern. Einerseits liegt dies daran, dass die APN bislang in der Schweiz kein geschützter Titel ist und somit auch das Handlungsfeld auf Institutionsebene individuell definiert wird. Dies kann zu Rollenunklarheiten und Verunsicherungen führen und den Nutzen immer wieder infrage stellen. Andererseits müssen auch die finanziellen Mittel für diese Stellen zur Verfügung gestellt werden. Insbesondere auf politischer Ebene ist viel Überzeugungsarbeit nötig, um die Etablierung der Rolle in der Schweiz voranzutreiben. Ein wichtiges Argument, um die verantwortlichen Personen von dem Nutzen der APNs zu überzeugen, ist sicherlich das positive Feedback von Patienten bzw. der Eltern, aber auch die positiven Patientenergebnisse, die durch pflegewissenschaftliche Forschungstätigkeiten belegt werden können. Jedoch sind APNs gefordert, hier durch Forschung und Leistungsausweis ihre Arbeit in den verschiedenen Kontexten aufzuzeigen. Das persönliche Ziel der eigenen Rollenfindung konnte mit dem Workshadowing mehr als erreicht werden. Die Erstautorin wurde in ihrem Wunsch bestärkt, ihr Fachwissen für Familien kardiologischer Kinder einzusetzen.

\section{$\rightarrow$ LITERATUR}

1 Walker RE, Gauvreau K, Jenkins KJ. Health-related quality of life in children attending a cardiology clinic. Pediatric Cardiology 2004, 25(1): 40-48

2 Hamric A, Spross J, Hanson C. Advanced Practice Nursing: An integrative approach. 4th Ed. St. Louis: Saunders; 2009

3 Wilkinson JD, Sleeper LA, Alvarez JA, Bublik N, Lipshultz SE, Group PCS. The Pediatric Cardiomyopathy Registry: 1995-2007. Progress in Pediatric Cardiology 2008, 25(1): 31-36, DOI: 101016/j.ppedcard.2007.11.006

4 Wilkinson JD, Landy DC, Colan SD, Towbin JA, Sleeper LA, Orav EJ, Lipshultz SE. The Pediatric Cardiomyopathy Registry and Heart Failure: Key Results from the First 15 Years. Heart Failure Clinics 2010, 6(4): 401-413, DOI: 10.1016/j. hfc.2010.05.002

5 International Council of Nurses. (2002). Definition and characteristics for nurse practitioner/advanced practice nursing roles [official position paper]. Download unter http://www.icn. ch/networks_ap.htm.PH/2002, letzter Zugriff 25.08.2015

\section{DANKSAGUNC}

Bedanken möchte ich mich ganz herzlich bei Gaby Stoffel für die interessanten Einblickstage in ihre wertvolle Arbeit.

\section{E AUTOREN \\ Gabriela Staub \\ $R N, M S c N$ cand. an der Zürcher Hochschule für Angewandte Wissenschaften in Winterthur. (ZHAW). Schwerpunkt ihrer Arbeit ist die pädiatrische, \\ kardiologische Pflege im Universitäts-Kinderspital Zürich. \\ E-Mail: staubgab@students.zhaw.ch

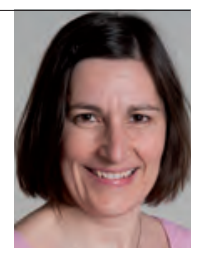

Dr. rer. medic.

Daniela Händler-Schuster Dozentin MScN und Projektleiterin des Instituts für Pflege an der Zürcher Hochschule für Angewandte Wissenschaften in Winterthur (ZHAW). Schwerpunktthemen sind unter anderen das Leben mit Beeinträchtigungen im Alter sowie die Professionalisierung der Pflege. E-Mail: haed@zhaw.ch

Prof. Dr. Romy Mahrer-Imhof Professorin für familienzentrierte Pflege an der Zürcher Hochschule für Angewandte Wissenschaften in Winterthur (ZHAW). Sie leitet den Studiengang Master of Science in Pflege. Sie setzt sich seit Jahren für APN ein und unterrichtet zu Rollenentwicklung der Advanced Practice Nurses in verschiedenen Studiengängen.

E-Mail:mahr@zhaw.ch

\section{BIBLIOGRAFIE}

DOI 10.1055/s-0041-109614

JuKiP 2016; 1: 10-14

(c) Georg Thieme Verlag KG

Stuttgart · New York · ISSN 1439-2569 